\title{
New Understanding in Academic Science
}

\author{
Joseph J. Smulsky
}

Institute of Earth's Cryosphere, TyumSC, SB RAS, Tyumen, Russia

Correspondence to: Joseph J. Smulsky, jsmulsky@mail.ru

Keywords: Outworld, Methodology, Knowledge, Interactions, Public Problems

Received: November 2, $2018 \quad$ Accepted: March 15, $2019 \quad$ Published: March 18, 2019

Copyright $\odot 2019$ by author and Scientific Research Publishing Inc.

This work is licensed under the Creative Commons Attribution International License (CC BY 4.0).

http://creativecommons.org/licenses/by/4.0/

\section{(c) (1) Open Access}

\section{ABSTRACT}

The present paper is a compilation of extractions from author's works. Those works were performed along different lines of research, each line reflecting some relevant side of the surrounding world. The review is focused on the world around us and on our understanding of this world. While writing the present paper, author tried to outline the disadvantages of the contemporary scientific picture of the world. Those disadvantages mainly stem from the presently adopted scientific method in which almost all explanations of physical phenomena are based on hypotheses. Gradually, the arbitrariness of adopted hypotheses gets forgotten, and fictional explanations get establish as the real structures of the world. In the present paper, the electromagnetic and gravitational interactions are treated from the position of the no-hypothesis approach. The latter approach consists in the study of phenomena as they are, in the measurement of the various properties of natural objects, and in the establishment of relations between them. The gained results form a new understanding of the world's phenomena which will allow using them in humans' practical activity. Moreover, the new understanding of the world permits anticipation of future results without recurring to hypotheses. Some unveiled sides of the surrounding world are also analyzed, together with the problems the human society presently faces. In conclusion, author summarizes the main shortcomings of the contemporary academic science which have caused public distrust in obtained results. Author would like to emphasize here that science can only earn trust in society via reliability and trustworthiness of its reached results. Author hopes that this publication will prove interesting for a wide range of readers.

\section{INTRODUCTION}

In contemporary academic science, both in foreign and domestic, or, in other words, in the mainstream science, some system of views has established. This system of views is a self-sustaining one due to the adopted practice of publication of scientific works. According to the adopted rules, every work must 
pass a peer-view procedure organized so that the merits of the work could be evaluated by some Mainstream participants, and only if approved by the reviewers, the work can be recommended for publication on the condition that it conforms to the whole system of the currently adopted views. The authors of published works receive grants and prestigious awards, and enjoy honor. Their activity is considered successful, and it is the way that the scientific research in academic institutions goes.

However, there are many scientists, and other literate and inquisitive people, who distinctly see the shortcomings of the established concepts and, sometimes, even glaring contradictions and absurdities resulting from them. In my opinion, I myself belong to such people. This year marks the 50th anniversary of my work in academic science. There were years when I worked in design bureaus or applied research institutes, but my work on the fundamental science never interrupted.

The present work was written by me as stimulated by the Russian Literary Center, whose staff members suggested my participation in the contest "Great Ideas". I was invited to present my concepts along three directions: statements, quotations and fragments. I have therefore prepared some extractions from my previous works arranged along these three directions. When I had systematized my compilation, I saw my work presenting a self-consistent system of views free of the shortcomings intrinsic to the mainstream science. Scientists doing research in academic institutions often expressed their agreement with many of my thoughts. In turn, in the views of other scientists I saw the same understanding at which I have arrived. Therefore, the understanding of the surrounding world suggested in the present paper is a new understanding that has originated and exists currently in academic science alongside with the mainstream understanding.

Both in Russia and abroad, we often express our dissatisfaction with the mainstream science, or academic, science. On the one hand, the understanding of the micro- and macro-world offered by this science is incredible. On the other hand, the grounds for such understanding are rather ephemerous and unreliable.

According to the established rules, within the framework of academic science any new understanding of the surrounding world should be based on facts. The understanding of the world described in the present paper is such an understanding.

I sent the first version of my paper [1] to the journal "Herald of the Russian Academy of Sciences" at the beginning of October 2017. Then, I believed that the new understanding in science could become a trump card for rehabilitation of science in society. But I was wrong, and my paper was rejected. The arguments delivered by the editorial staff in regard of my paper are outlined in p. 65. After reading the present paper, the reader will also receive the opportunity to evaluate the presented concept as it looks for people who share traditional views, i.e. from the side of the old understanding.

How should this paper be read? It contains brief statements, many of which were borrowed from my much more extended works. Therefore, each of these statements has reliable justification. Here, nothing is said for nothing and in vain. Here, everything is on the case. If anyone puts something to doubt, let him check for himself the fact that has caused a controversy in him and then, I am sure, he will also become convinced of my truth.

As I have already mentioned, in the present publication my thoughts are expressed in concentrated form, i.e. in the form of a concentrate, like e.g. the concentrate of hydrochloric acid. In strongly diluted form, the latter acid facilitates digestion, but in concentrated form it burns everything through. Therefore, the paper should be initially read without haste. You need to think: maybe something is wrong here, not presenting the final truth? Then, after getting acquainted with what follows, you may feel needing to return to some of the previous points in order to see if you happened to encounter a contradiction here.

This paper is not intended just to make your time busy with reading. A properly spent time is a time during which a person gains knowledge.

All the extractions from my works are sequentially numbered, and each is supplied with reference to the original source at the end of the paper. The fragments are arranged in ten sections. The title of each section points to the problem to which particular samples belong. Many of the problems are so vast that they by no means can be exhausted by the presented content. 


\section{THE SURROUNDING WORLD AND ITS PERCEPTION}

1) Everything consists of two: the world around us and our understanding of this world ([2], p. 8).

2) The idea of the world and the real world. Let us turn to Homer. Here Achilles throws a spear to kill Trojan Aeneas, the son of Anchises; yet, piercing the shield on its periphery, the spear gets stuck in the ground. Achilles exclaims with surprise:

"Gods, here is a great miracle done before my eyes!

There lies my spear on the ground, and not a trace can I see

Of the fellow I meant to kill, whom I wished to overthrow!

Truly, this Anhiside enjoys the favor of Olympic deities!"

Achilles sees the reason for saving from the inevitable death of Aeneas in the action of the gods. This is not a metaphor or literary device invented by Homer. Reading the ancient authors, we see that gods invisibly lived among people, governed the wind and rain, gave light to the Sun and Moon, directed people and controlled their actions. Now we consider such ideas very naive. We know what the air and its composition are, under what conditions the air takes on speed and becomes a wind or even a terrible hurricane, and so on. Our knowledge is so reliable that we without hesitation consider the views of the ancient people about the world highly erroneous.

In this connection, some questions arise. Are our own ideas about the world always true? Which of them will appear rejected by our descendants and considered naive? Are there ideas of ours that will remain never discarded? Can we identify our misconceptions based on faulty thinking or understanding? If so, then let us identify them and discard them for ourselves; then we will not bring ourselves to shame before future generations.

Around us is the surrounding world,- the sky, the stars, the trees, our house, the objects in it, and so forth. These objects of the surrounding world are changeable, affecting each other. We study these objects, find ourselves explaining the reasons for the observed changes that occur to those objects and, in this way, create our own understanding of the world. If, in ancient times, the forces of gods or demons produced an explanation to the world, nowadays we explain the world with the aid of forces, fields, ether, energy, space-time, and other similar notions. As we see, any explanation and any understanding of the world are also things that undergo permanent changes, while the world itself remains largely unchanged ([3], p. 277).

\section{CRITERIA OF KNOWLEDGE OF THE SURROUNDING WORLD}

3) What is truth? The truth is that, guided by what, we achieve what we aspired to. [June 1, 1992].

4) What is the action (or influence)? Under the action of one object upon another object we understand the ability of the former object to set in motion the latter object or to change its motion. To change the motion of a body-this means to change its velocity either in magnitude or direction, i.e. to give this body acceleration ([2], p. 36).

5) It should be noted that the "field" is a mathematical term introduced to indicate the dependence of a function on the coordinates. In nature, there are no fields as people invented fields. In nature, there is an action of some objects on other objects. To explain this effect and visualize it, the concept of a field was introduced ([4], p. 40).

6) Everything consists of two: the world around us and its description.

The world around us is a part of everything that does not depend on the way of our thinking and reasoning.

Description of the surrounding world is the understanding, or interpretation, of the idea of the surrounding world, i.e. its representation.

Knowledge is an understanding of the world around us such that it will not undergo significant changes with time.

Truth is knowledge by using which we find results of our actions complying with our intentions.

Under the truth, we usually understand some fundamental item of knowledge which plays an important role either always or at a certain moment. 
Science is the field of human activity aimed at gaining new knowledge about the world around us.

Theory is a description of the properties of the world's objects, the methods of human activities, and their results.

Time is a quantity that characterizes the variability of objects and is perceived by comparing the changes that occur in objects with the changes of a reference (standard) body/object.

The time of existence of an object, or its lifetime, or the duration of a process, is the number of the cycles of the standard change being equivalent to the change in the phenomenon/object/process under consideration.

The time in an object's change results from binding a certain stage of this change to a certain stage or cycle of the reference change.

The time interval between two different stages of an object's change is the number of the cycles of the standard change that have proceeded between these stages.

Mathematical time is the result of the comparison of changes in objects with a stable cyclic change in an imaginary standard.

Mathematical time is used in the theoretical description of the surrounding world.

The size of an object/body is the result of a comparison performed by superimposing some standard on the object; this size is expressed as the number of standards or the number of parts of the standard that can be superimposed onto the object.

The size of the object is determined along three mutually perpendicular directions which in decreasing order are called the length, the width and the thickness.

The name of the object's size can be linked to the vertical dimension (height/depth); to the horizontal dimension (width/thickness); or to the sides of the world (latitude, longitude, etc.).

The size of the gap between objects is the result of a comparison performed by placing standards in between objects; this size is expressed as the number of standards or their fractions.

The sizes between objects are arranged in three mutually perpendicular directions.

The sizes of the intervals are called distances.

Space is the total of the objects and the gaps between them.

This physical definition of space should be distinguished from the word "space" used in mathematics, poetry, fantasy and other areas of human activity ([2], pp. 240-242).

\section{METHODS OF GETTING KNOWLEDGE ABOUT THE WORLD AROUND US}

7) The non-hypothetical approach consists in studying phenomena/objects, in measuring their properties, and in establishing dependencies between both. All this needs to be done in order to comprehend the circumstances under which phenomena occur. Then, on the basis of the dependences obtained, a description is created, which can be called the theory of the phenomena. The theory allows the prediction of phenomena and using them for the benefit of the man ([4], p. 7).

8) All our knowledge of the real world is built on a set of words/concepts. Some of those words/concepts are the names of objects in the surrounding world, while others are the names of some mental images created by the man to describe the surrounding world... Apparently, both the language and the knowledge of the world have been created in the process of human evolution and, therefore, there is no clear distinction between the two kinds of concepts. Very often, mental images and even objects of human imagination were perceived as objects existing outside the person in the real world.

The notions of space and time are typical ones in this respect. I. Newton had formulated them as some absolute and unchanging notions to be used to describe the phenomena in the surrounding world, implying that in all considerations the same rectilinear space and the same uniformly flowing time are to be used. Subsequent generations transformed the Newtonian notions of space and time into the names of some categories of the real world, having made the imagined world the real world. The theory of relativity had rejected the absolute space-time and introduced a new amorphous concept of four-dimensional space-time. I. Newton never considered the absolute space and time as some essences of the surrounding 
world; equally, the relativistic picture of the world had emerged just as a (very illogical) representation of the real world but not the real world itself (note: something relativistic is something based on the theory of relativity).

Thus, the difference between the real and the unreal must be comprehended already at the stage of invention of words and concepts. If a person realizes that one type of words is necessary for denoting some thing in the surrounding world, and another type of words, for inventing a description (perception, explanation, representation) of this thing, then the problem of reality immediately ceases to exist.

Below, we give some examples concerning this point.

a) Is the mass a measure of the amount of matter?

And what is mass?

The mass of a body (see [4], Sections 1.2 and 4.2) is the number of standard bodies that, under the action characterized by some acceleration, stretch the spring by the amount same as that the body does when imparted with the same acceleration.

And what is matter?

Matter is a common name for all objects in the surrounding world (a tree is a common name for oak, pine, cherry, etc.; nonmetal is the general name for wood, plastic, sand, etc.; substance is the general name for metals, nonmetals, liquids, gases, etc., and so on until a complete generalization of the surrounding world with the word "matter" is reached).

Answer: Matter cannot have quantity/measure.

b) Is there a god in the world?

And what is god?

God is a mythical being which controls the surrounding world.

The answer is: there is no god in the world (see the above definition of the notions "god").

c) Does the ether carry light?

And what is the ether?

Ether is...?

Answer: the question is posed incorrectly.

It is clear from the examples given above that, with a clear definition of notions, the problem of reality never arises. What is required is only to use notions by their strict definitions... Like it is impossible to include an electric bulb rated for $3.5 \mathrm{~V}$ in $220 \mathrm{~V}$ network, a notion cannot be used under circumstances that even very little differ from the circumstances that appear in the definition of the notion. The consequences in the latter case are more serious than those in the former case ([4], pp. 254-256).

9) Comprehension of the two kinds of concepts is necessary for the passage to the no-hypothesis investigation of nature. Nowadays, the highest assumed achievement in scientific activity is the development of a theory. They say: hypotheses were accepted, a model for a phenomenon was constructed, and a theory was developed. This strategy itself creates a picture of the world. Since such a conception of the world is based on speculation, in the process of further study of natural phenomena this concept comes in conflict with facts and, then, it must necessarily be discarded. The task is to gain knowledge about the real world that would remain unchanged in time, such as Newton's law of gravity, Coulomb's law, Faraday's law of induction, Newton's laws of mechanics, etc. The above laws allow one to describe, calculate and predict natural phenomena. An analysis of those laws shows that some of these laws are fully conditioned by the methodical approach like, e.g. the laws of Newtonian mechanics (see [4], Sections 1.2 and 4.2), whereas other laws appear as a result of measuring some properties of natural objects. On elimination of all model mechanisms of interaction from the existing methodical description, the essence of the no-hypothesis approach will appear looking as follows.

Among the whole variety of interacting bodies, pairs of bodies are to be distinguished whose interactions are typical of the interactions of many bodies, e.g. the interaction between the Earth and the Moon or the interaction between charged objects. Interactions between reference bodies (e.g. between a platinum cylinder (kilogram) and the Earth) are to be introduced for each type of interaction. Therefore, the description of any type of the interaction between other bodies will require the determination of the number 
of equivalent reference interactions. Next, in the reference interaction the magnitudes of forces are to be measured for different distances between the bodies and, if the force of the interaction depends on the relative velocity of bodies, also for different relative velocities. Thus, the obtained dependences will allow one to perform a correct calculation of the actions and predict the results of the actions. In this way, by introducing some standards of certain properties (such as the standards of length, mass, time, heat capacity, and light intensity) and comparing the properties of the bodies of interest with those of the standards, we get the true knowledge of the world. Of course, the fragmentarily no-hypothesis approach presented here cannot be expressed in the form of some strict rules or axioms. But this is the way which we undoubtedly must follow ([4], pp. 256-257).

10) With the proper use of statistical methods, you can justify any thing, and anything can be refuted ([5], in html format, p. 10).

11) It is often said that, without the use of hypotheses, it would be impossible to comprehend the surrounding world. At the same time, it is believed that the initially adopted guesses and presumptions can be put to test, and those presumptions which we failed to confirm, discarded. Then, there will remain guesses and presumptions that are consistent with the observed phenomena.

An analysis of the process of human-brain work and thinking shows that this is mere self-deception. The temptation to succumb to the magic influence of a bright hypothesis arises in a person when some details/circumstances escape notice or remain obscure to him. You can adopt the hypothesis immediately; yet, it may take years until all the details/circumstances will be comprehended. But an experienced specialist will not allow himself to be misleadingly carried away by a hypothesis. He will continue his difficult and hard work on the study of objects, their properties, the influence exerted on the objects under study by other objects and by relevant circumstances. As a result, he will achieve such an understanding of the objects under study that he will be able to foresee and describe their behavior without invoking hypotheses ([2], p. 24).

12) In my life, I have solved many mathematical problems... then, in the declining years, I heard that my colleagues talked about me as of a skilled mathematician. Later, I began to realize that the problems solved by me probably would probably have not been solved by other people.

The fact is that, to tackle all solved problems, I used simple elementary operations. And in those times it seemed to me that anyone familiar with elementary and higher mathematics could easily perform those operations and, in this way, solve the problem. It turned out, however, that this is not so. Very often, some of scientists cannot learn and use in their work the ready solutions I obtained. The reason for that is that, in solving the problems, I use, instead of the modern mathematics, another calculus. To show in which respect the alternative mathematics of mine differs from the traditional mathematics, it is necessary to consider particular examples of solved problems ([6], p. 10).

13) In my letter to Chan Rasjid [7], I wrote that I had been creating a new physics, without the theory of relativity. For creating a new physics, the contemporary mechanics and mathematics are not appropriate. Mechanics is spoiled by the too abstract Lagrange and Hamilton methods, and mathematics, by the curvilinear geometry and set theory ([6], p. 18).

14) I returned to the methods of the 19th century and improved those methods. Contemporary mechanics and mathematics were developing in the 20th century under the influence of the theory of relativity. Yet, science had not benefited much from that development ([6], p. 18).

\section{DISADVANTAGES OF THE CONTEMPORARY SCIENTIFIC UNDERSTANDING OF THE WORLD}

15) The contemporary scientific understanding of the micro- and macro-world is false. Many of its objects are imaginary, and they do not exist. Since the beginning of the 20th century, science has established as a method to describe the surrounding world on the basis of hypotheses that were put forward with the aim of achieving an adequate understanding of the world. Based on those hypotheses, a theory is created to describe and explain phenomena. Then, the invented fictional explanation is perceived as the 
actual structure of the world ([4], p. 4).

16) As a result, the modern scientific picture of the micro- and macro-world has been constructed around a set of sequentially adopted presumptions. With passage of time, the picture moves farther and farther from the reality and, because of the contradictions existing between the hypotheses, the minds of the scientists professing the validity of those hypotheses get confused. On the one hand, those scientists are unable to get out of this imaginary world. On the other hand, they remain unaware of their being in a wrong belief ([4], p. 4).

17) Over the period of past two centuries, mechanics and mathematics have evolved under an irresistible desire of scientists to generalize their results and methods, and to make those results and methods more and more abstract. In teaching new generations, the genesis of concepts is often omitted. Therefore, the majority of modern physicists share the opinion that it is notions of the ultimately high level of abstraction that present real aspects of the surrounding world, with measured quantities being just consequences of those notions ([4], p. 46).

18) In modern physicists, the thought process proceeds only within the sphere of ultimate abstraction. As a result, the theories logically deduced within abstract concepts are only considered in the space which many physicists cannot escape.

Only those physicists who recognize Nature as the primary thing, and our methods to describe the Nature, the secondary tricks, can get out of the vicious methodological circle ([4], p. 47).

19) I would like to put forward the following ultimate thesis: the contemporary science is defective and false...

Why is contemporary science defective and false? The contemporary science is defective and false because that science has created an unreal and fantastic picture of the micro- and macro-world, and because it does not oppose the anti-social and anti-human tendencies in the modern world thus contributing to the degradation of mankind ([5], p. 8).

20) The theory of relativity has created in science a set of false goals. Among such goals are the search for gravitational waves, the manipulations with "black holes", the various models of the universe, and some theories in micro-world physics on which many scientists work. It was proclaimed that common sense... could not be a criterion of truth. The violation of common sense and logics in paradoxes of the theory of relativity (TR) is now perceived by many people as a feature being intrinsic to all modern theories ([4], p. 45).

21) The false hypothesis of the dependence of mass on velocity has led people to the false constructions in the micro-world. One of such constructions is neutrino ... now neutrino is introduced into all nuclear reactions. The energy balances of those reactions were experimentally measured. The experimental values were increased by theorists by the fictitious neutrino energy, and then those values were immediately reduced by the same amount of energy because all neutrinos always fly away unhindered ([4], pp. 8-9).

22) Relativists, or supporters of TR, usually assert that the TR has been confirmed by experimental facts and observations. However, this is not true. Moreover, the TR conclusions contradict the practice. After all, the so-called TR paradoxes come as the conclusions of a theory that contradict the practice and all practical activities of people. As many scientists showed in the past and show today, all the phenomena that were calculated using TR can also be calculated with the same success without using that theory ([4], pp. 220-222).

23) The theory of relativity has had a profound impact on our understanding of the world, on our worldview, and on the basics of our knowledge. In many discussions, one can hear time and again from the TR supporters that the relativistic law of addition of velocities is the exact law, whereas the simple summation of velocities in classical mechanics is an approximate law valid only for the case of low velocities. When we showed that the classical summation of velocities is identical to the arithmetic action $2+2=$ 4 while the relativistic equivalent of that equality is $2+2=5$, we were answered roughly the following way. Both the first and second summation laws are hypothetical, but the relativistic law is confirmed experimentally, and so it is correct ([2], p. 10). 
24) Contemporary science holds and lives on great sensations. The last sensation was the detection of gravitational waves. The previous sensation concerned the discovery of the Higgs boson, God's particle, on the supercollider in Switzerland. At this collider, according to its creators, the Big Bang is modeled, as a result of which our Universe has arisen. Before that sensation, there was a sensation about possible collision of the Earth with the asteroid Apophis ([5], p. 9).

25) An example of the latest sensation and its elementary checks. In their paper, B.P. Abbott and his 1100 co-authors have described results of their observation of gravitational waves emitted as a result of a collapse of a double Black hole. This is the so-called LIGO experiment, where LIGO is the abbreviation for the name of the observatory that was established for the detection of gravitational waves by laser interferometer (Laser Interferometer Gravitational-Wave Observatory).

On September 14, 2015 at 9:50:45 the employed device had recorded a pulse with several oscillations, which lasted about 0.15 seconds ... the fusion of the black holes had imparted the test body with acceleration of $10^{-21} \mathrm{~cm} / \mathrm{sec}^{2}$.

The first point. The reader can imagine that the equipment used to perform the LIGO experiment should register test-body displacements as small as $1.1 \times 10^{-23} \mathrm{~cm}$. The diameter of the hydrogen atom is $0.529 \times 10^{-8} \mathrm{~cm}$. Therefore, it can be said that, for performing the LIGO experiment, an apparatus was created that allows measuring lengths constituting $2 \times 10^{-15}$ fraction of the atom size.

The second point. The collision of the black holes and their merging together had occurred at a distance of 410 megaparsecs. This distance was determined with an accuracy of -180 to +160 megaparsec. If expressed in light years (l.y.), this distance is 1.34 billion l.y., lying somewhere in the interval from -0.59 to +0.52 billion l.y.

Since gravitational waves propagate at the speed of light, the fusion of black holes could happen within a time interval from 590 million years ago till 520 million years in the future (!).

The third point. The collision of two objects of size $D$ moving at velocity $v$ occurs in a time $t=D / v$. The pulse from their collision will have approximately the same duration $t$. For example, a collision of two bullets normally occurs in a time of $t=60$ microseconds, and the collision of two ocean-going liners, in a time of $t=0.5$ minutes. Such will also be the duration of the collision-generated pulses. Consider now the collision time of two galaxies, with black holes residing at their centers. The standard diameter of galaxies is $D=10^{5}$ l.y., and their relative velocity is $V=1000 \mathrm{~km} / \mathrm{sec}$. We will take into account the fact that one light-year is equal to $9.461 \cdot 10^{12} \mathrm{~km}$. Then, the collision time can be evaluated as $t=30$ million years.

So, the duration of the pulse produced by two merging galaxies moving at a relative velocity of 1000 $\mathrm{km} / \mathrm{sec}$ is $t=30$ million years, and not $t=0.15 \mathrm{sec}$, which time was registered in the LIGO experiment.

Suppose that LIGO scientists are right, and they have indeed detected a collision of two black holes. Let us evaluate the velocity of the colliding black holes; for this velocity, we obtain a value $v=D / t=21,000$ billion. $c$, where $c$ is the speed of light.

That is, in the LIGO experiment, the galaxies moved at velocities exceeding the speed of light by twenty-one thousand billion times.

The theory of relativity is based on the fact that nobody can move faster than light. The publication of the LIGO experiment was timed to coincide with the 100th anniversary of the general theory of relativity to allegedly confirm this theory.

Evidently, the LIGO experiment has in fact disproved the theory of relativity. Therefore, based on this "discovery of the century" in the mainstream science, once again I say: the theory of relativity and the entire modern physical picture of the micro- and macro-world must be thrown away and forgotten! ([5], pp. 9-10).

26) Over a period of 10 - 15 years, Stephen Crothers, a researcher from Australia, in his numerous papers based on four-dimensional curvilinear geometry have been arguing that the expanding universe, the Big Bang, gravitational waves and Black holes all were impossible constructions. The general theory of relativity was developed using the same geometry. Visually and wittily, Stephen Crothers had expounded his results in his lectures, and his audiences responded to him with stormy applauses. Over all those years, there has not been a single relativist who had at least somehow challenged Stephen Crothers' arguments. 
Why? Because that in the world of academic science nobody understands both the special and general theories of relativity. Nevertheless, at the general meeting of the Russian Academy of Sciences which was held on March 22-23, 2016, the gravitational-wave sensation had resounded as a milestone scientific discovery, and plans for creating a national collider for reproduction of the Big Bang were announced ([5], $\mathrm{p}$. $10)$.

27) In my refutations published in [5], I confess, I used references to my papers and papers by other authors published in little-known journals. This is clearly my fault: for almost 50 years, all the well-known journals rejected my papers on this topic. However, the guilt of other opponents of the theory of relativity is even greater: their papers have been rejected by all known journals for one hundred years ([5], in html format, p. 10).

\section{ELECTROMAGNETIC INTERACTIONS AND THE SPECIAL THEORY OF RELATIVITY}

28) I have derived expression for the force. It follows from this expression that at zero relative velocity of bodies the force is determined by the Coulomb law. With increasing velocity, the magnitude of the force decreases, and at a velocity equal to the speed of light, the force vanishes. Thus, not mass, space and time change with the motion of the body; instead, it is the force of its electromagnetic interaction with other bodies that undergoes changes ([4], p. 8).

29) Light and radio waves represent the electromagnetic influence of one body upon another. The properties of the propagation velocity of this interaction are defined by the laws mentioned above of electromagnetism. These properties differ from the properties of the velocity of bodies. The lack of understanding of many optical experiments on light propagation between moving bodies is due to this difference ([4], p. 9).

30) The theory of relativity embraces a significant field of science, including physics, philosophy, mathematics and methodology, on which the numerous predecessors, creators and followers of this theory have piled up so many interrelations that the corresponding relationships have become inaccessible to logical analysis ([4], p. 35).

31) If we assume that in the case of rest and in the case of motion the interactions should be described by same expressions, then the parameters at rest can be expressed in terms of the parameters in motion. By itself, this method is quite permissible providing that we understand its conditional nature. But in TR that method was absolutized, and nowadays it is widely believed that, on the passage from rest to motion, the distance, time and other parameters all undergo changes ([4], p. 42).

32) At the end of the XIX century it had become clear that the interaction of charged bodies depends on the mutual motion of the bodies. G.A. Lorentz put forward a hypothesis according to which all bodies change their sizes during the motion, which circumstance in turns leads to a change in the force of interaction. This was justified by the ideas about the ether. Particles of matter, supposedly consisting of ether, while moving in the ether, can change in size. However, the interaction of two bodies does not depend on any absolute speed, but it depends on their mutual velocity. A. Einstein had modified the hypothesis of G.A. Lorentz and, as a result, he invented a description of the interactions between moving bodies in the form of interactions occurring between motionless bodies, whose parameters, the space, time and mass, varied according to the Lorentz transformations. This was the first line in the development of electrodynamics around which the contemporary physics was constructed. The second line was based on the finite value of the propagation velocity of the interaction. As a result, the interaction of two bodies moving with respect to one another is not equal to the interaction of stationary bodies at the same distance between them. It is believed that the fields expressing this effect act with a delay in time. If the fields that describe the interaction of stationary bodies are shifted in time by an interval necessary for the interaction to propagate between the bodies, then these fields will express the interaction with respect to moving bodies. Oliver Heaviside derived expressions for the field strengths in the electromagnetic interaction of bodies and extended these expressions to gravitational interactions. Later, this line was continued by O.D. Jefimenko. The method of delayed fields (or retarded potentials) can completely replace the Special and Gen- 
eral Theories of Relativity (SRT and GRT). As an alternative to the above two methods, I have developed a method resting completely on the mechanics. As a result of the performed analysis of the development of electromagnetism, I have arrived at the conclusion that the force of interaction of charged or magnetized bodies moving relative to each other depends not only on the distance between the bodies but, also, on their relative velocity. I have derived an expression for the force, based on the experimental laws of electromagnetism. Then, I have extended this method to other cases. A number of new problems have been solved, and new results were obtained, which have shown that our world is not the one that appears in the TR physics. This method is ready for use and my book [2] offers its detailed description ([2], pp. 9-10).

33) Conclusions

a) Light is the electromagnetic influence of the source upon the receiver.

b) The electromagnetic influence of one on another body depends on the mutual velocity of the bodies. This influence does not depend on the velocity of the receiver or source with respect to any imaginary environment.

c) There is no any luminiferous medium (ether, field, the medium of physical vacuum, etc.), the movement of the source or receiver relative about which would change the influence produced by the source upon the receiver.

d) The change in the frequency of light and in the direction of its propagation under conditions of the source's motion with respect to the receiver obeys the laws of electromagnetism ([8], p. 1607).

34) The essence of the principle of relativity consists in that there should be no changes due to the uniform and rectilinear movement. A classical example of this principle is usually the cabin of a vessel or the compartment of a train moving at a constant velocity. If the windows are closed and tightly sealed, and if they are provided with a screen impenetrable for electromagnetic waves and electrical interaction, then one can agree with such a formulation of the principle of relativity that none experiments can be used to determine whether the cabin is moving or not. But when the objects surrounding the train are visible through the windows, when an air stream passes through the compartment, when the light of some source or a radio wave is received in the cabin, and when there are charges or currents in the compartment that can interact with outside charges or currents-in that case any of the above phenomena can be used to identify the motion of the observer and, also, determine the velocity and direction of this motion ([4], pp. 74-75).

35) The essence of the TR obstacle is the following. In nature, there is an objective mutual influence of bodies. Electromagnetic interactions depend not only on the distance between bodies, but also on their relative velocity. The description of the interactions in TR is constructed in such a way that the equations for the interaction of mutually stationary bodies and the equations for the interaction of bodies in relative motion are the same. Therefore, in order to satisfy empirical data, it is necessary to transform parameters at rest to parameters in motion using the known relativistic transformations.

If the interactions between moving bodies are described as they are, that is, dependent on the velocity of the relative motion of bodies, then the transformations of space, time and mass are no longer required. This is the first point.

Secondly, the creators of TR inspired by the ether conception, obviously deceived themselves, believing that they had constructed not a description of interactions but a world in which the material bodies underwent changes by relativistic relations. And since relativistic transformations became imaginary at superluminal velocities, then the superluminal motions were banned in TR ([2], p. 245).

36) The theory of relativity is based on the following two erroneous assumptions:

a) The action of one moving material system on another such system does not depend on the velocity of the relative motion, and therefore for any velocity the equations should be written identically;

b) The relative velocity of moving objects cannot exceed the speed of light.

With the help of classical mechanics, electrodynamics and mathematics, equations were transformed and the theory of relativity was invented to satisfy the above two presumptions. Later, the theory of relativity was developed with the help of the TR apparatus, and in its consequences it had finally arrived at the 
denial of its starting presumptions, as well as at various paradoxes. Since paradoxes could not be eliminated from this theory, and the method of this theory has allowed many phenomena to be described, TR views had gradually established according to which the then-existing knowledge of people about things and relations in nature was concluded to be incorrect, together with the non-relativistic mechanics and electrodynamics ([4], p. 83).

\section{GRAVITATIONAL INTERACTIONS AND THE GENERAL THEORY OF RELATIVITY}

37) The hypothesis that the gravity propagates at the speed of light was introduced in order to make applicable, in treating the gravitational interactions, the false hypotheses stating that the mass, the length, and time come as quantities dependent on the velocity of motion of gravitationally interacting bodies. An additional justification of those hypotheses was allegedly obtained while considering the rate of Mercury perihelion's precession. Yet, taking into account the Sun's oblate shape explains all motions in the Solar system, including the rotation of Mercury's perihelion ([4], pp. 9-10).

38) The hypothesis of the expanding Universe contradicts the common sense if prolonged into past. With this hypothesis, it turns out that, initially, all objects in the Universe were separated with distances smaller than the sizes of those objects. This is an absurd. Meanwhile, this absurdity can be eliminated on adoption of the "Big Bang" hypothesis ([4], p. 5).

39) Everything that happens on the Earth and in the observable Universe is explained by the mutual attraction of objects governed by the Newton law of gravity. In this case, if the spreading of the objects would have begun, then the velocity of the objects would have inevitably been a decreasing function of the distance from the origin. Why? Because for any object the force by which this object acts upon other bodies will be directed toward the center of the aggregate, i.e. against the object's velocity. This will inevitably result in a decrease of that velocity ([4], p. 6).

40) For justification of the radially growing velocity profile of the expanding universe the "dark energy" was introduced. The purpose of this energy was to create repulsive forces acting to overcome the forces of gravitational attraction between objects and to make the objects more and more accelerated as they move farther from the point of Explosion ([4], p. 6).

41) One can imagine a star for which the second cosmic velocity is equal to the speed of light. If we accept the hypothesis of light consisting of particles, then we can readily deduce that such particles cannot overcome the gravity of the star, and for a distant observer this star would represent a dark body, i.e. a "black hole". In the case of gravity's propagation with the speed of light, a body would not affect the particles of light, and those particles would fly away from the star to the observer unimpeded ([4], p. 10).

42) According to the General theory of relativity, a ray of light passing near a star, e.g. the Sun, must move along a bent trajectory. This is possible if the light consists of heavy particles and the gravitational force obeys the Newton law. If the gravity propagates at the speed of light as suggested in the General theory of relativity, then the effect of the star on a particle moving at the speed of light would have never been observed, making the particle moving rectilinearly without curving its path ([4], pp. 10-12).

43) For confirmation of the General relativity, three phenomena were considered: the precession of Mercury's perihelion, the deviation of light trajectory in the vicinity of a gravitating body, and the change in the frequency of light passing in the vicinity of a gravitating mass. In my opinion, in the consideration of such phenomena the following strict rule must be adhered to: a groundless statement should never be put to confirmation. The only "basis" for GRT was the desire of its inventor to create a Unified field theory. But the world around us never adjusts to the desires of people.

Suppose that at constructing a theory we can ignore the above-formulated rule and consider this desire as a basis. The next statement of General relativity is that the gravity propagates at the speed of light. This idea has been continuously put to test since the formulation of Newton's law of universal gravitation. And every time it was rejected when a more precise solution of the differential equations of motion was obtained or the additional influence due to a previously ignored body was taken into account. Most complicated calculations of the gravitational interaction, those of the Moon' motion, were performed. On the 
basis of those calculations, in 1787 Laplace had arrived at the conclusion that, had the speed of gravity be finite, then it would exceed the speed of light by 100 million times.

Apparently, I have analyzed the whole chain of GRT allegations-and I have arrived at the conclusion that all hypotheses adopted in GRT had no firm empirical basis. In my works, I gave an analysis of presumptions adopted in GR, and now everyone who wishes to perform a similar analysis, can independently verify my conclusions ([3], p. 280).

\section{OTHER COMPONENTS OF THE SURROUNDING WORLD}

44) What is the essence of the impossibility to exert motion exclusively at the expense of internal forces? Why such a motion is impossible not only from the standpoint of classical mechanics? The answer is quite simple. One body or a set of bodies (a mechanical system) can start moving only if some other body acts upon this body or the set of bodies under consideration [9].

45) There exist problems whose solution largely determines the further path of mankind. One of such problems is the problem of two bodies. As a result of the solution of this problem for gravitational interaction, an understanding of why and how bodies move in the outer space was gained. Under the influence of the Sun, a planet moves around it almost in a circular orbit, and a comet, along an elongated elliptical or, sometimes, along with a parabolic trajectory. For directing a spacecraft to Mars, it is necessary that, relative to the Earth, the spacecraft would travel in a hyperbolic orbit.

The understanding of such things and all related human activities would have been impossible if the two-body problem was not solved. Many people fail to understand this. There are people, even scientists, who believe that planets revolve around the Sun due to the vortex rotation of the ether, with the Sun being its sink (or source). The ideas expressed by such people take their origin in the Descartes hypotheses. Now we can imagine what wrong path the mankind would have taken if the problem of two bodies was not successfully solved by I. Newton.

In this paper, we consider solutions of three problems on the interaction between N-bodies. In my opinion, the impact of those problems, and their solutions, on the future development of mankind is comparable in significance with that due to the solution of the problem of two bodies ([10], in html format, p. 2).

46) In a vortex chamber (Figure 1), a medium, e.g. swirling air, moves from the peripheral regions of the chamber toward the central hole provided in the upper cap. Then, the air leaves the vortex chamber moving upward. As the medium approaches the chamber axis, due to the conservation of the angular momentum, its tangential velocity increases, goes through a maximum and, then, vanishes as the moving medium runs up to the chamber axis. Near the axis of the vortex, large tangential velocities may result even if the velocity of the air the chamber's periphery remains comparatively low. For instance, the maximum tangential velocity of the medium in the vortex chamber can exceed the peripheral velocity by 45 times [11].

The pressure falls along the radius of the vortex chamber toward its axis and, at high air velocities, rather large pressure drops develop. In tornadoes such pressure drops present one of the most destructive factors. For instance, if the pressure at the tornado centerline falls to $90 \mathrm{kPa}$ and the tornado runs into a building in which atmospheric pressure $(100 \mathrm{kPa})$ is maintained, then a load of 1 ton is applied to each square meter of the building surface, making the building explode. Simultaneously, there may be objects near the destroyed building upon which the tornado will produce no equally destructive effect.

At the centerline of the vortex (see Figure 1), there forms a counterflow with an air stream diverging from the centerline [11]. It is the flows that create the cyclone eye. In some cases, at the centerline of tornadoes, objects depressed into the earth surface such that, e.g. a pressed layer of straw from a scattered haystack or tree branches stuck in the ground, can be found. This effect can be due to the counterflow. The velocity of the counterflow, as it adds to the velocity of fall of the objects in the field of gravity, will lead to the objects' penetration into the soil. Strong electrization of the rotating bed of particles in the vortex chamber is observed, and electrical discharges occur there, both processes bearing close resemblance to the 


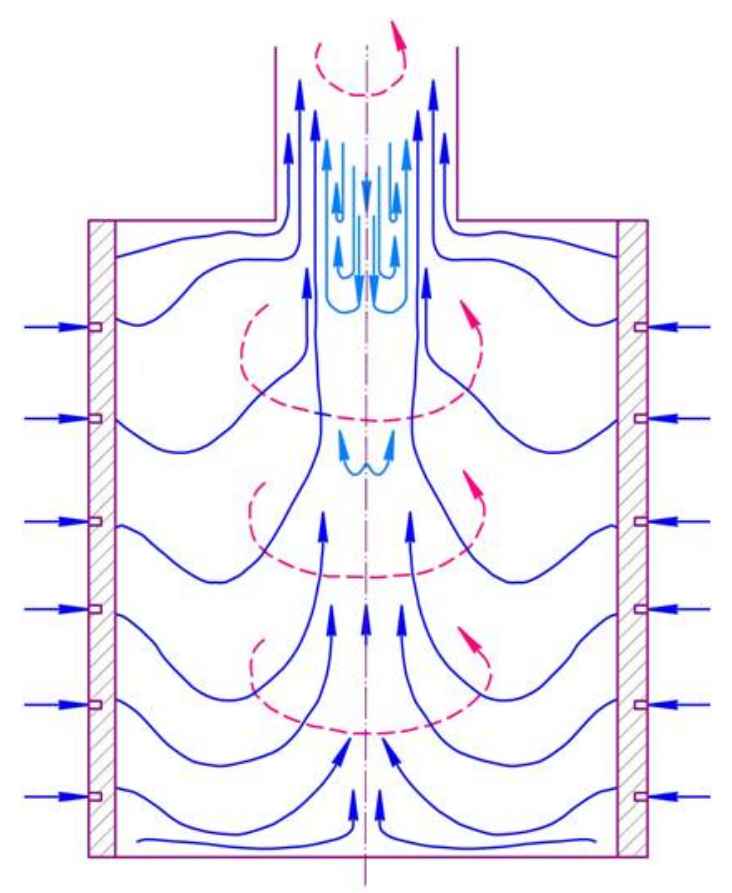

Figure 1. Flow pattern in a vortex chamber with a peripheral injection.

electrical phenomena occurring in tornadoes (lightning flashes traveling through the tornado channel and radio waves) [12].

In the vortex chamber, the rotating medium moves to the centerline. A similar mechanism of formation of rotating funnels operates when the water sinks out of a reservoir. Atmospheric vortices are formed in the same way: more buoyant air strata superheated on the surface ascend upward through the flow core, this core being smaller in diameter than the draining region. This ascent will persist unless the superheated air stratum gets exhausted. Unlike in a reservoir, here the sinking point and, hence, the atmospheric vortex, both can move relative to the earth's surface.

In the vortex chamber, the pre-rotated swirling flow is normally generated at the periphery of the chamber due to tangential air supply. When the liquid or the atmosphere undergoes sinking, the initial swirling flow forms due to different factors [11]. In the vortex chamber, the draining of the medium proceeds due to the drop of pressure between the inlet and the outlet, whereas in a reservoir, such draining is induced by gravity, and in atmosphere, by buoyancy forces ([1], pp. 14-15).

47) In our opinion, efficient measures for combating the intense atmospheric vortices can only be taken with due consideration given to the formation mechanisms of such vortices.

Apparently, posing the problem of control for small atmospheric vortices, that is tornadoes, is presently quite possible. For preventing the formation of a tornado, the possibility of realization of a low-speed inflow of air from the near-ground zone should be ensured. For this purpose, it would be necessary to create such vertical impulse that it would ensure the formation of either one large channel or several smaller channels acting as sinks for the air. With the sink having been created in advance, the superheated air will not be accumulated in the near-ground zone; instead, it will permanently ascend upward at a low velocity.

Thus, the draining of the near-ground air into the vortex and its upward motion inside the vortex are not to be suppressed here; instead, both are stimulated with the aim of preventing the accumulation of the energy of air inside the near-ground zone with the danger of subsequent catastrophic release of this energy. For creating the vertical impulse, it is necessary to deliver some portion of fuel to a certain height, to spray the fuel there, and ignite it ([1], p. 38). 
48) On September 22, 1991, at 10 a.m. I flew by flight No. 239 Moscow-Tyumen that was carried out by a TU-154 aircraft at an altitude of $11,000 \mathrm{~m}$. The bright Sun was shining in misty atmosphere, with snow-white clouds being seen below. Below and on the right I saw a brightly sparkling disk that accompanied us. The disk had clearly defined edges, sometimes it grew clouded, with its boundaries getting broken, and rays emanated from it. The disk followed the plane, sometimes lagging behind it and then catching up with the plane. I recalled numerous publications describing the cases when aircrafts were accompanied by flying saucers. The disk I observed had the same properties as those that were described in the publications I read.

My observations lasted from 10:15 till 10:40. Time to time the disk flared brightly like the Sun and grew a bit dim; during the aircraft's yawing in flight, the disk lagged behind the aircraft and then outstripped it synchronously with the Sun, Then, the disk disappeared in the clouds and, sometimes, fragments of lakes or rivers appeared in its place. Then, the clouds had completely disappeared at the bottom, and it had become clear that it was the Sun that was observed reflected along the sight line of the disk from all the water surfaces on the ground. The disk was nothing else then the reflection of the Sun from the upper boundary of the clouds. This reflection differs from the reflection of the Sun in the mirror or in a water surface. The latter reflection can be observed at any angles of the Sun's elevation over the reflecting surface, whereas the reflection from clouds can only be observed at a certain angle.

Measuring the distances and constructing the line of reflection and observation had shown that the Sun was at an altitude of $22.5^{\circ}$ above the horizon. If at this time the course of the aircraft were perpendicular to the sight line of the Sun, then the passengers looking in the portholes would observe a UFO (unidentified flying object), which in fact was the Sun's reflection. Such observations can be lasting long in the case of homogeneous clouds for an aircraft flying to the West (in that case the angle of the Sun's observation above the horizon changes little). On moonlit nights, under such conditions the Moon's reflection will be observed ([13], pp. 14-15).

49) When I hear various religious programs on the radio and judge the manner in which those programs reflect the world by communicating the listeners some religious ideas about it, this makes me deeply indignant.

The contemporary man has studied many things in the surrounding world. He knows the whole surface of the Earth, and what this surface consists of. He knows the composition of the environment: air, water, soil. He has studied the structure of the Earth. He has studied almost everything around him. He has divided the environment into a living and non-living nature. He has studied what the living world consists of, how it develops, how it had originated, and how the inanimate nature was changing over the past millions and billions of years. He understands what the sky is and what are the stars, what is the Sun and what the Moon is... Man has established that the planets move around the Sun. Now, we definitely know that there exist other innumerable planets like those we observe in our Solar system. Each star, in principle, is surrounded by such planets. We also know that there is an even greater world than the world around the Sun, in which each star is among other stars like a grain of sand in the desert. We call this world a galaxy. There is even a world of a greater level, in which the galaxies themselves appear as grains and form meta-galaxies. Man has learned what matter consists of, and he has revealed the tiniest substance particles.

And then the Christian religion tells us that 6000 years ago there was a word, and this word was God, and within a few days the whole world was created. One can find such ridiculous tales offered to modern mankind rather upsetting. Indeed, what path is to be taken by the society? How can we prepare our younger generation for their future lives? All the knowledge gained by mankind must be conveyed to this generation and be taught by young people.

Along with those tasks posed, today the younger generation in our country is spoiled with a completely different picture of the world, and with quite different ideas. How can an individual be guided, how can he act and exist in the world?

Education of a person, and education of his thinking, is like writing some programs and putting them in a computer. Imagine now that you use a program to determine actions that the computer must perform 
in order to solve a problem. Then, you run another program using which you determine completely wrong, false, and absurd actions. Will such a computer work?

And we feel surprised looking at the contemporary young man. He has perfectly false, logically contradictory, and completely fantastic ideas about the world put in his head. Simultaneously, we want this person to do logically and rationally in the surrounding world. This is a task for psychologists: what actions such an individual will undertake? Either he/she will reject the false ideas internally, or he/she must alienate herself from the real world and will be guided by myths. Or, probably, he/she will prefer choosing a combined strategy using in some situations real knowledge while adhering to religious knowledge in other cases? Under such circumstances, many people will become mentally and psychologically disabled and they simply will not be able to perform their vital tasks and take proper actions for orientation in the surrounding world ([14], p. 2).

50) Every person is born and lives in the world. From time to time, we encounter some turning points, or events that happen to each of us. A person passes from child- to adulthood, this process being a most important event for the individual. Then he establishes a household and family: he finds a person of the opposite sex, which becomes his second half in the proceeding life. Then he gives birth to his children, and after him his offspring remains. Then the person goes out of life. These five events are momentous ones, important for any person, and they must be approved by the society in such a way that every person should be aimed at pursuing the natural course of life. Therefore, these important events in the life of each person must be sanctified by the society.

There must be some holy things in the human society, thanks to which people who do not know what is good and what is bad will follow the natural course of life. If not, then many people who do not know what to do, what is allowed and what is forbidden, and cannot be transgressed or violated, will act in such a way that chaos will establish in the society. Therefore, there must be certain rules for a living individual by which he must be guided in life.

Of course, there must be sacred places sanctified by society, but not under control of some ruling person. For any individual, the sacred objects are his monument, his family, his things, and when he gone, the next ruler may say that those objects are not holy at all, and people will scatter them on the ground and trample them with their feet. The sacred objects of the society should be identified by its best representatives who are not rulers but who have deserved the respect of people through their activities during their life ([14], p. 2).

\section{PROSPECTS FOR FURTHER DEVELOPMENT OF SCIENCE AND HUMANITY}

51) We are on the verge of revolutionary changes in physics. The logic of common sense will prevail, the mysticism will disappear from the notions of time and space, the classical mechanics will take its deserved place, and great achievements in our understanding of the macro- and micro-world will occur. Philosophy and methodology will get rid of fetters, and science will provide us with the true knowledge, simple and clear like the four arithmetic actions ([4], p. 47).

52) You are right, the special and general theories of relativity are obviously wrong. These theories need to be thrown away and forgotten. Erroneous are also all fields of physics in which the theory of relativity is used.

I am engaged in creating a new physics without the theory of relativity; this physics uses no Lorentz transformations and curvilinear geometry. Join this work!

You are also right in your believing that mathematics is needed for solving physical problems. However, contemporary mathematics and mechanics are not suitable for the new physics. Mathematics is spoiled by the set theory and by curvilinear geometry, and mechanics, by the too abstract methods by Lagrange and Hamilton.

I work to update mechanics and mathematics. I have solved many problems, including the problem of Mercury's perihelion [15] [16], and have developed many new research methods. I have provided free access to these results to all interested scientists. 
I have created a powerful tool for comprehending the micro- and macro world. This tool is the Galactica system. It is designed to solve the problems of interaction of bodies under the laws of Newton and Coulomb. Sample tasks and instructions for the Galactica system are freely available.

I appeal to people making my proposal to them to use the obtained knowledge for gaining a better insight in the world and thus to create a new physics.

Proponents of the fake physics prevent publication of my works while the published works are being suppressed. Those people also try to block access for interested scientists to my sites, so, please, have time to copy the materials and data unless the sites are closed.

As for the book by Florentine Smarandache et al. "Unresolved problems in the Special and General Theories of Relativity" (by Florentin Smarandache et al. http://www.worldsci.org/php/index.php?tab0=Books\&tab1=Display\&id=1641), there are no unsolved problems in the Special and General Theories of Relativity except for one: these theories need to be thrown away and forgotten!

The book "Unsolved problems in the Special and General Theories of Relativity", written along the Theory of Relativity, is a useless and false book. In evaluating all works on physics, I suggest using the following criterion: "Physical theories, which are not based on the works by J.J. Smulsky, or which contain no mention of his works, are false and deserve no attention!" [7].

53) The existence of solutions according to which a sphere of a radius equal to the Earth orbit's radius can accommodate several Earth-like planets rotating around the Sun opens up new prospects for further development of mankind. New planets can be created from the substance available in the Solar system. This is the substance of asteroids, that of the planets' satellites, and even the substance of the planets themselves. New planets can be created sequentially. First, a second planet after the Earth is to be created. That planet is to be inhabited with primitive forms of life and, after the rooting of those forms, we can further proceeds by introducing more evolved plant and animal species on the created planet. This process is to be continued until conditions suitable for human life will finally establish on the planet. From this moment on, a longer stage of spreading the humans and the entire living world over the new planet will begin.

On the Earth, life has originated from multiply repeated random interactions. Therefore, the process of its origin and evolution had taken billions of years. As a result of purposeful human activity, it can be hoped the same process on the new planet will be completed in several hundred, and not billions, years.

After assimilation of the second planet, works on the creation of the third, fourth, etc. planets can be launched. With the assimilation of every new planet, the orbits of all other planets are to be adjusted in such a way that to allow the existing planets perform regular motions according to the exact solution of the $3 \mathrm{D}$ problem for the given number of bodies.

To create a new Earth, mankind needs to gain experience in handling large space objects. In 2008, a proposal was made by us to turn the asteroid Apophis into an Earth satellite. On April 13, 2029, this asteroid will approach the Earth to a distance of six Earth's radii, and there will be no more such close approaches during the next one thousand years. It is desirable that the satellite Apophis should revolve in the same direction in which the Moon does. For this, six months before the approach the Apophis needs to be accelerated slightly to order to make it moving on the night side of the sky. The retardation then will become required at the moment when Apophis will approach the Earth.

Another scheme was proposed for the transformation into satellite of even larger asteroid 1950 DA. To make that asteroid rounding the Earth's orbit, it should be accelerated slightly at the point of its aphelion, and then retarded at the moment of approaching the Earth. In this way, we will be able to make the 1950 asteroid a satellite rotating around the Earth in geostationary orbit.

Making the asteroids into Earth's satellites are simpler operations than the creation of a new Earth. However, those operations are currently technologically unfeasible. The proposal for Apophis advanced in 2008 for the year of 2029 had a time reserve of 21 years. With purposeful work that could be done during 21 years, the mankind could accomplish even more cardinal tasks. For example, after the devastating war, the Soviet Union in 12 years had launched a satellite, and in 16 years it launched a manned spaceship. 
These were accomplishments made by just one country. Now we can imagine what accomplishments could be achieved by the whole mankind in 21 years if all countries unite their efforts ([10], p. 16).

54) As a result of my work on the problem noted in Section 4 [17], I have created a regular spherically distributed structure of $N$ bodies, which for $N=100$ bodies is shown to remain stable after 100 revolutions.

The reviewer has accused me of my not proving the fact that the system would also remain stable after $10^{7}, 10^{8}$, and $10^{9}$ rotations. It should be noted here that until now there is no proof, except for mine for $10^{8}$ rotations, of stability of a Solar system involving ten bodies, the Sun and nine planets. Therefore, this accusation is ridiculous.

I will give you another example. The first railway in Russia was built in 1837 to connect St. Petersburg and Tsarskoe Selo. Luckily, there were no such reviewers in those times who could blame the road builders for not building a railway around the Globe. Imagine now that such reviewers would have been, and the tsar listened to them. Then, there would be no railways thus far in Russia.

Reverting to regular spherical structures, I express my confidence that, with time, all things will be proved, and new Earth-like planets, created. This will be done notwithstanding the fact that there exist such reviewers, and there exist those who blindly believe them. But, simultaneously, there are people like me, although few in number, who carry knowledge and truth which will be inevitably accepted by all rationally thinking people.

At this point, the reviewer expresses doubt about possible methods which will allow mankind to solve the problem of creating Earth-like planets. It is worthy to note that, apart from the difficulties mentioned by the reviewer, there exist many other problems within the proposed project, and those problems are clearly indicated in my paper. Nonetheless, my paper proposes a good way towards overcoming all difficulties, suggesting as a starting point the transformation of asteroids into Earth's satellites.

Unfortunately, the reviewer fails to evaluate my suggestions as he belongs to dogmatic scholars engaged in reading rather than in doing original research. On the contrary, my paper is written for creative scientists. Such scientists never evaluate scientific papers by looking for points in them to be blackened; instead, they try to see in papers new solutions deserving implementation ([17], p. 23).

55) In his General Comments, the reviewer describes his impression imposed on him by my paper "Advances in Mechanics and Outlook for Future Mankind Progress". This is an impression of a person familiar with ideal mathematical models, who treats such models in isolation from the surrounding world. He believes that the surrounding world functions obeying laws that will never become known to him.

On the contrary, in my paper I tried to show that the recent achievements in mechanics have provided an adequate explanation to all aspects of the surrounding world, thus opening new possibilities for the improvement of this world and further development of mankind. I have assigned my paper to the category of research papers since, in my manuscript, results obtained by most advanced methods are presented. Moreover, there results were tested for reliability in terms of all possible criteria at present.

The reviewer concludes:

1) the settled results of the "official" science are challenged by the Author's results and research.

2) among about 25 references, only three are not the Author's papers;

3) the paper does not bring any new result.

These conclusions obviously contradict each other. Indeed, if some results challenge the existing notions, then they are obviously new. On the other hand, truly new results have no analogues in literature, and in the latter case there is no need in making reference to anybody.

In conclusion, I state the following. The reviewer's opinion that my paper lacks new results is obviously erroneous. All the particular comments made by the reviewer reveal his bad command of logics and physics in treating physical problems. Since no signs of error in my paper were identified, this paper should be regarded as a paper deserving publication as it offers to the reader perfectly new, highly relevant, and quite interesting content.

I suggest that the journal editorial board has published my paper, together with the report provided by the reviewer and with my response to that report. 
Contemporary problems of mankind are largely caused by the crisis in the establishment, or mainstream, science. There is no High Arbitrator in the world telling people what is true and what is false. When two opposing opinions are presented to rationally thinking people, those people for themselves will be able to make the right choice. Such choice will allow the mankind to solve all challenging problems the world presently faces ([17], pp. 23-24).

\section{WAYS TOWARD THE DEVELOPMENT OF SOCIETY}

56) The formula of today's (1992) insanity: "The bifurcation of the presentation is funded by the stagnation of consensus" ([18], p. 163).

Or else: "Sequestering the legitimacy of investments is imitating by the mentality" (October 15, 1997).

57) At a dangerous time we live. The world is ruled by slander, lies and deception. This is the result of the activities the ruling circles of the United States of America conduct now. This policy was imposed on the Soviet Union and further practiced in Yugoslavia, Iraq, Libya, Syria and Ukraine. Previously prosperous countries have been destroyed. In the world, chaos was sown, confusion has established in the minds, and complete disorientation of the ruling circles has occurred in the leading countries ([19], in html format, p. 2).

58) The nuclear-weapon States have concluded a non-proliferation Treaty. But what about the states which have no such weapon? They are under the threat of the nuclear states. At the same time, the prevailing mass of weapons is possessed by the United States and by US-controlled states. The nuclear weapon of other states is ineffective against the US and their allies, since the development of such weapon is blocked by the US. Therefore, all states are under the threat of the US' nuclear weapon. If nuclear states retain the right of retaliatory strike, then nuclear-free states will be completely disarmed in the face of the atomic threat.

Therefore, there are no international and human rights prohibiting for the non-nuclear states independent of the US the creation of their own atomic weapon! Such weapon will be created secretly, and it will inevitably get in the hands of malicious people.

The US threat to the independent states is obvious and inevitable, and the atomic weapon is the only way to oppose this threat.

How can mankind get rid of nuclear weapons? Maybe mankind will be able to solve the problem in another way, via avoiding the threat of nuclear war? There can only be one option: all nuclear weapons must be controlled by all. No country should be able to use nuclear weapons at its own will. Only in that case all states will be feeling safe against the face of the nuclear war ([19], in html format, p. 2).

59) One of the main tasks for society for all ages was creative work. Signs of such creative work were found in England (Stonehenge, 7 thousand years ago), in America (Machu Picchu), in Egypt, in Asia Minor, India, China and in some other places.

The society should have as many achievements as all its members can make in total. What to achieve and what to do? There are two ways here. The first way is based on the technological development of society. Over the past 100 years, various discoveries were made that have brought to life new activities. Other new activities will appear in the future.

The second way is the activity aimed at the improvement and development of the world around us on the Earth. All previous activities were leading to the destruction of this world. Now the mankind's activities must pursue the purpose of the world's revival. The sentence "We will create a kingdom of life on Earth" should become the motto of society. In all places-plains, mountains, rivers, lakes and seas-the conditions for life must be cardinally improved... [2].

60) The contemporary residents of large cities, accustomed to entertainments and idleness, are unable, with rare exceptions, to follow the path of creativity. It is necessary to create a new capital of the state, in which people will start with their own hands building a life without all the shortcomings that exist in the contemporary society. The new capital should be located closer to the center of the state, for example, in the interfluves of the Ob' and Yenisei rivers. This capital should grow not upward, into the sky, in the form of skyscrapers, but in breadth. Modern means of communication and transport will allow people to live in 
separate villages and towns in which people will enjoy all the achievements of society and directly participate in the improvement and development of the environment. Both the capital and the adjoining settlements must fully provide themselves with food and material means, including energy.

When the creative goals of society are proclaimed, many young people, not only in Russia, but also in other countries, will rush to build the new capital. With their own hands, they will further detail these goals, clarify and develop them further. The peoples of the world are tired of idleness and inoccupation! Each person is created for labour, and the highest joy for him will be his achievements in work meant to bring benefit to all people ([19], in html format, p. 5).

\section{A THORNY PATH TO TRUTH}

61) We have arrived at a conclusion that many concepts in contemporary physics have emerged as a distorted description of the surrounding world. Much work remains to be done for correcting the errors and creating a new description. We hope that the new description of the surrounding world will be built on the no-hypotheses basis. The physical science will no longer be a collection of sacred hypotheses incomprehensible even for specialists. This science will give people a clear and explicit knowledge of the world, which will allow them to arrange their lives consciously, purposefully and rationally.

I am sure that there is nothing beyond the world and nothing inexplicable in the world. Yet there exist many things still unknown to us. I hope that, together with the Theory of Relativity, the mystical perception of the surrounding world will pass away, and the highest goal of society will be its desire for knowledge about the world (2, p. 244).

62) The attitude of English-speaking scholars to Russian authors is a serious problem. For example, such scholars treated me, a Russian-speaking person, as Brahmans treat sudra. Even the English-speaking Chinese researcher Chan Rasjid from Singapore had noted my Russian English which I tried to use while presenting to him my exhaustive explanations concerning the TR. Unfortunately, Chan Rasjid's understanding of the TR had stayed in the same place.

If the anti-relativists were closer to the reality and treated me as sudras treat a Brahman, then the TR would passed away 30 years ago [20].

63) What are the reader's benefits from reading this paper? Truth in life is important for a person, and truth in science is important for a scientist. The truth in life will only survive if scientists do not lose the truth in science. When the truth in life disappears, crashes occur in people's fates. However, the truth is still in science. Hence, there is truth in life. To find the truth and retain it, I suggest you reading my paper ([6], p. 18).

64) Statement of responsibility. For all the good and bad, true and erroneous in my work, I bear personal responsibility: I did it with zeal and without any evil intent. I did my job as I could, and who can, let him do better ([6], p. 38).

65) Before publication in "The Way of Science. International scientific journal" [1], the abridged Russian version of this paper was sent to the journal "Herald of the Russian Academy of Sciences". From the deputy Editor, on December 16, 2017 I received a decision letter rejecting my paper with the following motivation.

"Your paper was considered by the editorial board of the journal. Its publication is considered inadvisable, and no improvement or revision of the text can follow."

In the decision of the editorial board, it was noted that my reviewed paper in to Items $19,24,25$, and 52 was denying almost all contemporary achievements of science in the field of physics and space-physics. These items are reference to the papers published in the unknown journals "The Way of Science. International Scientific Journal" and "Natural Science".

Then, the editor proceeded as follows: "I think that these examples are quite sufficient. And the scientific world is unlikely to quickly follow the genius of J.J. Smulsky".

I acknowledge the awarding me with the title of "genius". However, I cannot so easily agree that the scientific world will never follow me. It already does, with my results being already in use. All other results 
will also find their applications, as these results were gained while studying natural phenomena coming from the surrounding world. They do not depend on erroneous presumptions or hypotheses, and they will always be true and fair.

\section{CONCLUSIONS}

The current understanding of the micro- and macro-world is based on a system of sequentially adopted hypotheses. The adoption of those hypotheses has led us to the construction of a world that does not exist.

The force of interaction between charged bodies depends on the distance between the bodies and on the velocity of their relative motion. The space, time and the mass of bodies all do not depend on the velocity of their motion.

The force of mutual attraction between bodies depends only on the distance between them. There is no good reason for ascribing a different nature to the force of gravitation. All hypotheses and their consequences about the expansion of the Universe, the Big Bang, gravitational waves, Black holes, Dark energy, etc. are false and should be discarded.

The contemporary academic science has created a halo of exclusivity around itself. This had allowed the science to create a fantastic world that cannot be put to doubt. This science has secured a system for publishing its works, financing them and setting the rating to those works in such a way that the research works criticizing the results of academic science did not develop and remain inaccessible both for society and academic science.

There is no reason for creating the halo of exclusivity around the academic science. Society will be able to assign a high status to science only when becomes convinced in the reliability of obtained scientific results.

All contemporary science must be analyzed in its very foundations. Weak bases must be discarded, and the consequences from them must be eliminated from science.

Society faces a lot of problems whose solution will only be found as a result of the examination of those problems. Without solving them, further development of society will be under threat. Society cannot entrust the solution of its vital problems to the contemporary academic science. Science can only earn trust of society considering the reliability of obtained results.

\section{ACKNOWLEDGEMENTS}

This paper is the result of the author's 30 years of work at the Earth Cryosphere Institute TyumSC of the Siberian Branch of the Russian Academy of Sciences, which in recent years has been carried out under the project IX.135.2.4.

\section{CONFLICTS OF INTEREST}

The author declares no conflicts of interest regarding the publication of this paper.

\section{REFERENCES}

1. Smulsky, J.J. (2018) The New Insight in Academic Science. The Way of Science, 1, 8-21.

http://en.scienceway.ru/archives

2. Smulsky, J.J. (1999) The Theory of Interaction. Novosibirsk: Publishing House of Novosibirsk University, Scientific Publishing Center of United Institute of Geology and Geophysics Siberian Branch of Russian Academy of Sciences, 293 p. http://www.ikz.ru/ smulski/TVfulA5_2.pdf (In Russian) http://www.ikz.ru/ smulski/smul1/English1/FounPhisics/TVANOT1.doc (In English)

3. Smulsky, J.J. (2007) Conceptual Error in Contemporary Science. Proceedings of the Natural Philosophy Alliance, 13th Annual Conference, 3-7 April 2006, University of Tulsa, Published Space Time Analyses, Ltd., Ar- 
lington, 3, 277-281. http://www.ikz.ru/ smulski/Papers/CnErCS2.pdf

4. Smulsky, J.J. (2014) Electrodynamics of Moving Bodies. Determination of Forces and Calculation of Movements. Palmarium Academic Publishing, Saarbrucken, 324 p.

http://www.ikz.ru/ smulski/Papers/InfElMvBEn.pdf

5. Smulsky, J.J. (2016) On Performance Indicator of Scientist's Scientific Work. The Way of Science, 10, 8-16. (In Russian) http://www.ikz.ru/ smulski/Papers/PokRezNauchD4.htm

6. Smulsky, J.J. (2015) Actual Mathematical Problems and Thorny Way of Science. The Way of Science, 10, 10-38. http://en.scienceway.ru/archives

7. Smulsky, J.J. (2015) Letter to Chan Rasjid. December 24, Tyumen, Unpublished. (In Russian) http://samlib.ru/s/smulxskij_i_i/rasjid01reendoc.shtml

8. Smulsky, J.J. (2014) Exact Equations for the Light Doppler Effect. Journal of Modern Physics, 5, 1602-1607. https://doi.org/10.4236/jmp.2014.516161

9. Smulsky, J.J. (2007) About Unsupported Movement. Unpublished. (In Russian) http://samlib.ru/s/smulxskij_i_i/obezopdvhtm.shtml

10. Smulsky, J.J. (2016) Fundamental Scientific Results and Habitat Formation of the Future Mankind. In: Drozdov, D.S. and Sadurtdinov, M.R., Eds., The Scientific and Industrial Activity Is Formation of Mankind Habitat: Materials All-Russian Youth Scientific Conference (with International Participation), 26-27 April, Tyumen, 10-18. (In Russian) http://www.ikz.ru/ smulski/Papers/FundScnRzl1.pdf

11. Smulsky, J.J. (1992) The Aerodynamics and Processes in the Vortex Chambers. Novosibirsk: Publishers "Science". 301 p. (In Russian) http://www.ikz.ru/ smulski/Aerpro/aerpro.djvu

12. Melnikov, V.P. and Smulsky, J.J. (1997) The Vortex Phenomena at the Atmosphere. Institute of the Earth's Cryosphere, Tyumen, 45 p. (In Russian)

http://www.ikz.ru/ smulski/smul1/Russian1/AtmVortex/VIHIAVL3.pdf

13. Smulsky, J.J. (1992) The Phenomenon of the Reflection of Celestial Bodies from Clouds and UFOs. Applied and Theoretical Questions of Non-Conventional Energy and Energy-Saving Technologies. Materials of the Scientific and Technical Conference, 28-30 September 1992, St. Petersburg, 24-27. (In Russian)

http://samlib.ru/s/smulxskij_i_i/ufo2html.shtml

14. Smulsky, J.J. (2004) The Radio "Word". About Belief and Nation Halidoms. Joseph J. Smulsky Answers the Questions of talk-Show Hostess Yu. A. Kerzhakov, Unpublished. (In Russian)

http://www.ikz.ru/ smulski/smul1/Russian1/Miscellena/VerSvNar.html

15. Smulsky, J.J. (2011) New Components of the Mercury's Perihelion Precession. Natural Science, 3, 268-274. https://doi.org/10.4236/ns.2011.34034

16. Smulsky, J.J. (2018) Future Space Problems and Their Solutions. Nova Science Publishers, New York, 269 p. https://novapublishers.com/shop/future-space-problems-and-their-solution

17. Smulsky, J.J. (2017) Advances in Mechanics and Outlook for Future Mankind Progress. International Journal of Modern Education and Computer Science, 9, 15-25. https://doi.org/10.5815/ijmecs.2017.01.02 http://www.mecspress.org/ijmecs/ijmecs-v9-n1/IJMECS-V9-N1-2.pdf

18. Smulsky, J.J. (1997) Nonrelativistic Picture of the World and the Ecology of the Mind (the Theory of Relativity versus the Mind. In: Malyushin, N.A., Ed., Engineer's Mind in Action, Giprotyumenneftegaz, Neftegazprekt, Tyumen, 163-175. (In Russian) http://samlib.ru/s/smulxskij_i_i/nerkar97.shtml

19. Smulsky, J.J. (2017) Improving the Abilities of Mankind to Further his Development. In: The Scientific and Industrial Activity Is Formation of Mankind Habitat: Materials of All-Russian Scientific-Practical Conference with International Participation, TIU, Tyumen, 29-36. (In Russian) http://samlib.ru/s/smulxskij_i_i/ssmdr02.shtml

20. Smulsky, J.J. (2017) A Letter to Harald van Lintel. 